\title{
MISMIDAD DE ACTO: ARISTÓTELES, HEIDEGGER Y ZUBIRI
}

\author{
ANTONIO GONZÁLEZ \\ Fundación Xavier Zubiri
}

\begin{abstract}
RESUMEN: Partiendo del análisis del empleo que hace Zubiri de la idea aristotélica acerca de la mismidad del acto (entre intelección e inteligido), el autor destaca el significado del complemento que el filósofo español propuso a la ontología de Heidegger: la idea de actualidad que está relacionada con el concepto de enérgeia. La diferencia entre actualidad y actuidad funge no solo para subrayar la concepción del ser como un momento ulterior de realidad sino para distinguir entre dos ámbitos: «ser presente» y «llegar a la presencia». En este punto, el artículo hace hincapié en el carácter invisible de los actos. Los actos, la enérgeia y el hypárchein no se refieren a la presencia de las cosas actualizadas sino a la actualización. En conclusión, el autor muestra que el modo como Zubiri vincula el análisis fenomenológico de los actos con la enérgeia conlleva las condiciones para una nueva antropología. En semejante contexto, la alteridad de la realidad no significa la formalidad de la realidad, sino la diferencia ontológica entre "surgir» y la cosa que surge.
\end{abstract}

PALABRAS CLAVE: actualidad, actuidad, hypárchein, enérgeia, ser.

\section{Sameness of act: Aristotle, Heidegger and Zubiri}

ABSTRACT: Starting from the analysis of Zubiri's use of aristotelian idea about the sameness of act (between intellection and intellected), the author points out the meaning of the completion that the Spanish philosopher proposed to Heidegger's ontology: the idea of actuality which is related to the concept of enérgeia. The difference between actuality and actuity is used not only to underline the conception of being as an ulterior moment of reality, but to distinguish between two areas: «being present" and "coming out to the presence». At this stage, the article insists on acts' invisible character. Acts, enérgeia and hyparchein don't refer to the presence of actualizated things but to actualization. At the end, the author shows that the way in which Zubiri links the phenomenological analysis of acts to the enérgeia involves the conditions for a new anthropology. In this context, the otherness of reality doesn't mean the formality of reality but the ontological difference between "arising" and the thing that arises.

KEY WORDS: actuality, actuity, hypárchein, enérgeia, being.

El estudio de la recepción por Zubiri del «acto» o enérgeia aristotélica tiene un punto álgido: el uso que Zubiri hace de la idea aristotélica de una mismidad de acto entre la intelección y lo inteligido.

Esta idea aparece justamente en el transcurso del diálogo de Zubiri con Heidegger, tal como lo encontramos en la última parte de Sobre la esencia ${ }^{1}$. Se trata de unas páginas fundamentales para entender los caminos divergentes emprendidos por ambos pensadores.

El marco general de este diálogo, en el libro de Zubiri, es la argumentación de Zubiri en favor de la prioridad de la realidad sobre el ser, en el contexto de su exposición del orden transcendental. Tras una referencia a la idea kantiana del

1 Cf. ZubiRI, X. (2008), Sobre la esencia. Madrid: Alianza Editorial, pp. 438-453. La primera edición es del año 1962. 
ser como posición, Zubiri señala que cabe otra comprensión del ser. No como posición, sino como un «dejar ser» (sein-lassen-von) ${ }^{2}$. Es una alusión directa a Heidegger, con el que inicia un diálogo.

\section{El Heidegger de Zubiri}

El diálogo con Heidegger comienza con una exposición del pensamiento del filósofo alemán, tal como Zubiri lo interpretaba en esos años. Hay algunas referencias inequívocas a varios de los textos de Heidegger que Zubiri utiliza en su interpretación. Además de Ser y tiempo, Zubiri alude a la conferencia sobre ¿Qué es metafísica?, la cual había sido traducida por el joven Zubiri al castellano, y que siempre desempeñó un papel importante en la interpretación zubiriana de Heidegger ${ }^{3}$. Y también encontramos referencias más o menos explícitas a otros textos posteriores de Heidegger, como Sobre la esencia de la verdad (1943) o la Carta sobre el humanismo (1947).

Si quisiéramos caracterizar la lectura que Zubiri hace en este momento de Heidegger, podríamos decir que el marco general de la interpretación zubiriana está determinado por la «comprensión del ser» como rasgo esencial del existente humano. Ello no significa que en la lectura zubiriana no haya ninguna presencia del Heidegger posterior a Ser y tiempo. Todo lo contrario: Zubiri desde muy temprano se dio cuenta de que la clave para interpretar a Heidegger no era ni el existencialismo, ni la hermenéutica, ni la ontología en el sentido usual de la expresión. El joven Zubiri entendía ya en los años treinta que el núcleo del pensamiento de Heidegger no estaba en el ser, sino en la verdad como característica del existente humano ${ }^{4}$. De hecho, ya en Ser y tiempo Heidegger llega a decir, a propósito de la verdad, que en ella «se da» (es gibt) el ser. Y esto significa entonces que este «darse» del ser, en que la verdad consiste, es algo tan originario como el mismo ser 5 . El desarrollo ulterior del pensamiento de Heidegger consistirá en afirmar la primeridad de la verdad respecto a la comprensión del ser como presencia, propia de la metafísica occidental.

Del mismo modo, la expresión «dejar ser», aparentemente secundaria en Ser y tiempo ${ }^{6}$, tomó relevancia en las reflexiones posteriores de Heidegger, tal como puede verse en el curso sobre Introducción a la filosofía, del año 1928-1929, al que asistió el mismo Zubiri ${ }^{7}$. Heidegger va centrando su interés en ese «dejar ser»

Cf. Zubiri, X., ibíd., p. 438.

3 Cf. Heidegger, M., «¿Qué es metafísica» (1933), Cruz y raya, 6, pp. 85-115.

4 Cf. Zubiri, X. (2002), Sobre el problema de la filosofía y otros escritos (1932-1944). Madrid: Alianza Editorial, p. 29n. Allí Zubiri cita el párrafo 44 de Ser y tiempo como criterio para interpretar todo el pensamiento de Heidegger.

5 Cf. Heidegger, M. (1986), Sein und Zeit. Tübingen: Max Niemeyer, p. 230.

6 Cf. Heidegger, M., ibíd., p. 354.

7 Cf. Heidegger, M. (1996), Einleitung in die Philosophie. Frankfurt am Main: Vittorio Kostermann. 
que, como tal, es anterior al ser. Sin embargo, Heidegger quiso evitar cualquier apariencia de subjetivismo. El «dejar ser», lejos de ser una iniciativa humana, es más bien una característica del ser mismo. Sin embargo, este ser ya no consiste en lo que está ahí presente, sino más bien en un sentido originario del ser (Seyn), que habría sido olvidado (por eso a veces Heidegger lo tacha) en la historia de la metafísica occidental. Sin embargo, en sus últimos escritos, Heidegger prefiere dejar de lado toda terminología explícitamente ontológica, y hablar simplemente del Ereignis como el acontecimiento en el que «se da» (es gibt) o "hay» ser ${ }^{8}$.

El hecho de que Zubiri aluda al «darse» como punto central de su discusión con Heidegger significa entonces que el pensador vasco, si bien no hace ninguna referencia explícita al Ereignis, era perfectamente consciente del rumbo que habían tomado las preocupaciones filosóficas de Heidegger. Dicho en otros términos: Zubiri bien sabía, a la altura de Sobre la esencia, que este "darse» del ser no consistía en una mera introducción a las cuestiones ontológicas en términos más o menos existenciales, al estilo de una «teoría ontológica del conocimiento». Todo lo contrario: Zubiri sabía muy bien que en este "darse» del ser se encontraba el núcleo del pensamiento de Heidegger ${ }^{9}$. Por eso Zubiri centra en el «darse» del ser su discusión con Heidegger.

\section{LA ESTRUCtURA DE LA DISCUSIÓN}

La discusión de Zubiri con Heidegger en Sobre la esencia se desarrolla en tres pasos. En el primero, Zubiri analiza el «dejar ser» como un «darse del ser». En el segundo paso, Zubiri afirma la prioridad de la realidad respecto al ser, acudiendo a la metáfora de la luz y del fulgor. Y en el tercer paso, Zubiri defiende la anterioridad de la aprehensión de realidad respecto a la comprensión del ser, para sostener que el ser humano no es "pastor del ser», sino "animal de realidades».

Normalmente, las exposiciones de las relaciones entre Zubiri y Heidegger se centran en los pasos segundo y tercero. Sin embargo, cada uno de los tres pasos de la discusión se funda en el anterior. En el primer paso, Zubiri muestra que el «darse» del ser ha de entenderse como una actualidad común entre el entendimiento y la cosa. Si esto es así, se puede mostrar, en el segundo paso, que esa actualidad común presupone la realidad de la cosa, de modo que el ser de Heidegger ha de considerarse como ulterior a la realidad. Y esta ulterioridad del ser respecto a la realidad presupone, tal como se argumenta en el tercer paso, que la aprehensión de la realidad es el presupuesto de toda comprensión del ser. De ahí que el primer paso sea el verdaderamente decisivo. Veamos esto más detenidamente.

\footnotetext{
8 Cf. Heidegger, M. (1969), Zur Sache des Denkens. Tübingen: Max Niemeyer, pp. 1-25.

9 Cf. Zubiri, X. (1962), Sobre la esencia. Madrid: Sociedad de Estudios y Publicaciones, p. 442.
} 


\section{El DARSE COMO ACTUALIDAD}

Resulta relevante que Zubiri se haya centrado en el «darse» del ser. Precisamente este aspecto del pensamiento de Heidegger es el que ha servido como punto de partida de las reflexiones sobre la donación que encontramos en autores como Derrida o Marion. No obstante, como es sabido, lo que en alemán se expresa literalmente como un «darse» (es gibt), también puede traducirse al castellano como «hay». Y la reflexión sobre el «hay» fue una de las vías exploradas por Zubiri en los años treinta para ir más allá del ser. Es un punto importante, que no vamos a tratar en este lugar. Sin embargo, Zubiri no habla aquí del «haber», sino explícita y literalmente de un «darse».

Zubiri entiende que el «darse» es una contribución especialmente valiosa de Heidegger. Lo que el «darse» expresa es que el «dejar ser» (o la «comprensión del ser») no constituye simplemente un acto en el que el ser se muestra a sí mismo y desde sí mismo. Además, la comprensión del ser, como «darse» del ser, es ella misma un modo de ser. Y por lo tanto es un modo de aquello mismo que se muestra. Es decir, el «darse» es un modo del ser ${ }^{10}$.

Y esto significa, para Zubiri, que Heidegger radicaliza el planteamiento fenomenológico clásico, en el cual el objeto de la comprensión sería algo así como un término extrínseco al acto mismo de comprender ${ }^{11}$. Por eso mismo, Zubiri acepta el planteamiento de Heidegger, afirmando que no quiere en modo alguno cuestionar esta tesis de su maestro, sino sólo completarla ${ }^{12}$.

Para completar el «darse» de Heidegger, Zubiri se dirige a los actos intelectivos, especialmente a la aprehensión como acto. Al hacerlo, Zubiri quiere evitar, frente a Heidegger, cualquier acusación de subjetivismo. Analizar los actos no es, para Zubiri, una vuelta a la «fenomenología de los actos de conciencia», porque los actos intelectivos no son tomados kathà dýnamin, sino kath' enérgeian ${ }^{13}$. Dicho en otros términos: Zubiri no quiere referirse a las potencias que, en el sujeto, hacen posible la realización de ciertos actos, sino a los actos mismos. Ahí es donde el análisis heideggeriano del «darse» ha de ser complementado.

¿En qué consiste este complemento de Zubiri? Aquí es donde Zubiri apela a la idea de actualidad, que se forjó en su filosofía justamente al hilo de su interpretación de la enérgeia aristotélica. Para Zubiri, en un acto de aprehensión, lo aprehendido no es sólo término del acto, sino algo que le confiere a ese acto su intrínseca actualidad concreta ${ }^{14}$. Lo que esto significa es que lo aprehendido en un acto no es algo extrínseco, sino intrínseco al acto mismo. El acto tiene entonces una actualidad concreta. Aprehendemos este sonido, y no otro; o este color, y no otro. El acto de aprehender está intrínsecamente configurado por

10 Cf. ibíd., p. 442

11 Cf. ibid., p. 443.

12 Cf. ibíd., p. 445.

13 Cf. ibíd., p. 451. Puede verse también ZuBIRI, X. (1980), Inteligencia sentiente. Inteligencia y realidad. Madrid: Alianza Editorial, p. 20.

14 Cf. ZubiRI, X. (1962), Sobre la esencia, op. cit, p. 443. 
lo que aprehende. Aprehender, en este sentido, no es solamente captar desde fuera, sino ser como lo aprehendido.

Esto significa que el acto es actual, que tiene una actualidad que le pertenece intrínsecamente. Ya en Sobre la esencia Zubiri distingue dos sentidos en los que se puede decir que un acto es «actual» ${ }^{15}$. El acto es actual por ser actualmente ejecutado. Y el acto es actual porque es el acto de una determinada actualidad intrínseca, en el sentido de ser el acto de aprehender una determinada cosa, y no otra. Años después, Zubiri establecerá una distinción terminológica entre los actos y su actualidad intelectiva o, dicho con la terminología final de Zubiri, entre la «actuidad»y la actualidad ${ }^{16}$.

Ciertamente, en el texto de Sobre la esencia, Zubiri todavía no distingue sistemáticamente entre "actuidad» y actualidad, sino que habla simplemente de dos sentidos de acto, o de actualidad, fundado uno sobre el otro. Una es la actualidad propia de la cosa, el hecho de que esté en acto, y otra es la actualidad intelectiva ${ }^{17}$. Esta indistinción terminológica, aunque no conceptual, tiene como consecuencia que Zubiri, en Sobre la esencia, todavía hable no sólo de una misma actualidad, sino también de un mismo acto ${ }^{18}$.

A la altura de Sobre la esencia, la unidad de acto, o de actualidad, consistiría en que, cuando aprehendemos algo, aquello que aprehendemos es una cualidad intrínseca del mismo acto. Así, por ejemplo, cuando oímos un sonido, la «sonidad» sería un momento del mismo acto auditivo. No habría, por tanto, dos actos, sino uno solo, que sería común a la cosa y a la mente ${ }^{19}$.

Esto no sólo sucedería en los actos sensoriales, sino que, según Zubiri, estamos ante un carácter propio de la noûs en general. La mente revestiría la cualidad formal, la actualidad, que es propia de las cosas. De ahí la profunda unidad entre la inteligencia y lo inteligido. Las cosas no sólo se muestran a nuestra mente, sino que «se dan», tal como Heidegger había señalado. Y, Zubiri añade, en este «darse», los sentidos y la inteligencia son en acto lo mismo que lo sentido y lo inteligido ${ }^{20}$. $\mathrm{O}$, dicho con la terminología posterior de Zubiri, los sentidos y la inteligencia tienen la misma actualidad que lo sentido y lo inteligido.

Esto no significa, sin embargo, que la mismidad de actualidad entre la mente y las cosas sea una mismidad de ser. En el caso del sentir, Zubiri indica, siguiendo a Aristóteles, que una campana puede estar sonando sin que yo esté oyendo el sonido. En este caso, el ser del sonido no tiene actualidad ninguna en la mente. En otras operaciones de la mente, como la de concebir un cuadrado, la mente no se hace cuadrada de una manera física, sino meramente «intencional». Por supuesto, en este caso, lo intencional no significa una correlación entre la mente y las cosas, porque entonces estaríamos volviendo a la idea de

15 Cf. ibid., p. 443.

16 Cf. ZuBIRI, X. (1980), Inteligencia sentiente. Inteligencia y realidad, op. cit., pp. 137-141.

17 Cf. Zubiri, X. (1962), Sobre la esencia, op. cit., p. 445.

18 Cf. ibíd., p. 444.

19 Cf. ibid., p. 444.

20 Cf. ibid., p. 444. 
un mostrarse extrínseco de las cosas a nuestros actos. La intencionalidad sería el modo de ser de las cosas inteligidas ${ }^{21}$.

En cualquier caso, lo que sucedería según Zubiri en todo acto intelectivo es que el «darse» de las cosas hace que la inteligencia sea lo mismo que lo inteligido, de modo que lo que se intelige no sólo se está mostrando, sino que está siendo. Esto tiene gran importancia para el diálogo con Heidegger. La mismidad de actualidad implica que la inteligencia se identifica con lo inteligido en todos los actos, y no sólo en aquellos en los que tiene lugar la comprensión del ser. Y esto significaría entonces que la peculiaridad del ser no habría que buscarla en el «darse», que es propio de todo acto, sino en las cosas que se dan.

Ahora bien, Zubiri es consciente de que lo que quiere decir Heidegger es otra cosa. La posición de Heidegger consistiría en decir que la índole del ser no es otra que ese "darse», propio de todo acto $^{22}$. Es lo que Zubiri analiza en el punto segundo de su crítica a Heidegger, donde nos habla de una ulterioridad de la «luminidad» respecto al fulgor propio de cada cosa, es decir, de una ulterioridad del «darse» (en que el ser consiste) respecto a la realidad. Sin embargo, ese punto segundo ya está decidido por los resultados del primero. La comunidad de acto entre la cosa y la intelección significa que la intelección está intrínsecamente configurada por la cosa, hasta el punto de que en la intelección no hay más acto que el de la cosa misma. La luz en la que se ven las cosas está configurada por el fulgor mismo de las cosas vistas.

La partida con Heidegger está entonces ya decidida. El camino desde la verdad, el dejar ser y el «darse» hacia el Ereignis no tiene por qué ser transitado. El verdadero «darse» es actualidad, y una actualidad que no es otra que la actualidad de las cosas mismas.

\section{La apelación a Aristóteles}

Zubiri entiende que este modo de completar el «darse» de Heidegger no es otra cosa que volver a ciertas «viejas ideas». Estas ideas son básicamente las de Aristóteles, aunque Zubiri incluye también algunas alusiones a la filosofía medieval ${ }^{23}$. Aristóteles, de hecho, sostuvo la comunidad de acto tanto respecto a los sentidos, como también respecto al conocimiento en general. Según Aristóteles «el acto del lo sentido y el acto del sentir es uno y el mismo, si bien su ser no es el mismo»" ${ }^{24}$. Del mismo modo, "lo mismo es la ciencia en acto que la $\operatorname{cosa} »^{25}$. De hecho, la discusión de Zubiri sobre el planteamiento de Heidegger en Sobre la esencia está hecho al hilo de estas citas de Aristóteles.

$21 \quad$ Cf. ibid., p. 445.

22 Cf. ibid., pp. 445-446.

23 Cf. ibíd., pp. 444-445.

24 ARistóteles, Sobre el alma, 425 b 26-27.

25 Ibid., 430 a 19-20. 
No es extraño que Zubiri pueda tomar las «viejas ideas» de Aristóteles sobre la comunidad de acto como punto de partida de su prolongación del «darse» de Heidegger. Como es sabido, las lecturas de Aristóteles, inspiradas por Heidegger, le sirvieron a Zubiri para ir más allá de Heidegger, iniciando su propio camino en el filosofar ${ }^{26}$. Y, de hecho, Zubiri, en esos años decisivos de su formación filosófica, siempre interpretó el acto aristotélico en términos de «actualidad».

Sin embargo, es importante tener en cuenta lo siguiente: Zubiri, en Sobre la esencia, expone sus ideas sobre la comunidad de actualidad usando a Aristóteles. En cambio, en Inteligencia sentiente subraya enérgicamente la diferencia entre su planteamiento y la idea aristotélica de una comunidad de acto. Lo que Zubiri quiere decir es que la comunidad de actualidad entre la inteligencia y la cosa no tiene que entenderse como una acción común producida por la cosa y por mi inteligencia. Esto sería una comunicación de sustancias, y por tanto una construcción metafísica, en lugar de un hecho accesible al análisis ${ }^{27}$.

Toda consideración sobre una posible comunicación de sustancias en una acción común se mueve, según Zubiri, en el plano de la «actuidad», y no de la actualidad. «Actuidad» es el carácter de acto, mientras que actualidad es el carácter de lo actual. El acto, en sentido aristotélico, sería según Zubiri «la plenitud de la realidad de algo ${ }^{28}$. Y, por eso, la actualidad de una cosa real se funda siempre en su "actuidad», es decir, en su realidad. Sin embargo, la actualidad no es la realidad, sino el estar presente de las cosas reales. La índole propia de la intelección sería justamente actualidad. La intelección sería actualidad de las cosas reales. Y la mismidad entre la intelección y las cosas no consiste entonces en una mismidad de acto, sino en una mismidad de actualidad. De este modo, la actualidad de la cosa real sería la misma que la actualidad del acto intelectivo ${ }^{29}$.

Esta idea de actualidad le permite a Zubiri, en su obra madura sobre la Inteligencia sentiente, mantener las líneas generales de su crítica a Heidegger. Cuando allí dialoga con Heidegger, Zubiri de nuevo no se dirige primeramente al ser, sino al «darse» del ser. En Inteligencia sentiente Zubiri habla concretamente de «desvelación», es decir, de la verdad en el sentido heideggeriano de alétheia. Y lo que afirma Zubiri es que toda desvelación es, en el mejor de los casos, una manera de actualidad, es decir, una manera en la que están presentes las cosas desde sí mismas como reales. Lo desvelado siempre sería algo que «está» desvelado. Y este "estar», esta actualidad, es un momento propio de la cosa misma. Según Zubiri es ella, la cosa, la que está presente desde sí misma como real. Y la intelección no sería otra cosa que este «estar». De nuevo entonces se afirma

26 Cf. GonzÁlez, A. (2008), «El eslabón aristotélico», Cuadernos salmantinos de filosofía Xxxv, pp. 5-36.

27 Cf. Zubiri, X. (1980), Inteligencia sentiente. Inteligencia y realidad, op. cit., p. 156.

28 ZuBIRI, X., ibíd. p. 137.

29 Cf. ibíd., pp. 134-141. 
la prioridad de la realidad de la cosa actualizada sobre su desvelación ${ }^{30}$. Dicho con la vieja metáfora: de nuevo nos encontramos con la prioridad del fulgor (realidad) de las cosas sobre su luminidad («darse»).

Aquí se nos plantean dos cuestiones, una de tipo histórico, y otra de carácter sistemático. Desde el punto de vista histórico, podemos preguntarnos por la índole de esta interpretación zubiriana del acto aristotélico. Desde el punto de vista sistemático, tenemos que averiguar si la comunidad de actualidad constituye un análisis satisfactorio de lo que constituye la esencia del acto intelectivo, de manera que, también desde la filosofía del último Zubiri quede obturado el camino hacia el Ereignis.

\section{El acto en Aristóteles}

Aristóteles, en su análisis de los distintos sentidos del ser, presenta varias listas de tales sentidos, diversas entre sí. Como es sabido, Brentano trató de sistematizar esta multiplicidad de sentidos del ente en una lista de cuatro: (1) accidente, (2) verdad y falsedad, (3) acto y potencia, y (4) el ser según las categorías, siendo la sustancia la primera de todas ellas $^{31}$.

Pues bien, Zubiri, en la mayor parte de sus exposiciones de Aristóteles ${ }^{32}$, recurre usualmente a una lista de los sentidos del ser que no coincide exactamente con ninguna de las listas aristotélicas, y tampoco con la lista de Brentano. De acuerdo a esta lista de Zubiri, el primero de los sentidos del ser sería la ousía como primera de las categorías, a continuación tendríamos el ser como accidente y el ser como esencia, en tercer lugar tendríamos el ser como acto y potencia, y finalmente tendríamos el ser como verdadero. A veces Zubiri, desde un punto de vista expositivo, «comienza por el final», como él mismo nos dice ${ }^{33}$. Esto significa que su presentación de los sentidos del ser en Aristóteles comienza por la verdad, es decir, por lo más cercano a nosotros, para terminar por la ousía, a la que Zubiri desde muy pronto traducía, no por «sustancia», sino por «sustantividad $»^{34}$.

Se podría decir que esta exposición zubiriana de los sentidos del ser está hecha, no al hilo de alguna de las listas de Aristóteles, sino más bien siguiendo

30 Cf. ibid., pp. 135-136.

31 Cf. Brentano, F. (1862), Von der mannigfachen Bedeutung des Seienden nach Aristoteles. Freiburg i. B.: Herder'sche Verlagshandlung.

32 Cf. Zubiri, X. (2007), Cursos universitarios, vol. 1. Madrid: Alianza Editorial, pp. 139-369; Zubiri, X. (2009), Cinco lecciones de filosofía. Con un nuevo curso inédito. Madrid: Alianza Editorial, pp. 11-55; ZuBIRI, X. (1995), Los problemas fundamentales de la metafísica occidental. Madrid: Alianza Editorial, pp. 39-69. p. 54 .

33 Cf. ZuBiRi, X. (1995), Los problemas fundamentales de la metafísica occidental, op. cit.,

34 Como se ve en el texto sobre Aristóteles en los Cursos universitarios, vol. 1, ya citado, y que procede de los años 1931-1932. 
el desarrollo general de la Metafísica de Aristóteles, tal como nos ha sido conservada. Sin embargo, hay que tener en cuenta que la verdad, como sentido del ser, aparece en la exposición aristotélica en dos ocasiones. En la primera, se señala que el ser verdadero no se da en las cosas, sino en el pensamiento, y que por tanto estamos ante un sentido secundario del ser ${ }^{35}$. Sin embargo, la cuestión queda abierta y vuelve a ser tratada por Aristóteles al final de la exposición de los sentidos del ser, cuando la verdad aparece considerada en el marco de la presentación del sentido del ser como acto $^{36}$. Y es que, para Aristóteles, la verdad puede ser considerada como un acto ${ }^{37}$.

Desde este punto de vista, se podría argüir que el ser como acto culmina la exposición aristotélica del los sentidos del ser. En cambio, en la exposición de Zubiri, el sentido del ser como acto no es el culminante, a diferencia de lo que sostendrían otros intérpretes actuales del Estagirita ${ }^{38}$. Lo que tenemos más bien es una exposición de Aristóteles que se encaja perfectamente en el marco de la filosofía de Zubiri. Tendríamos, en primer lugar, la ousía, y en segundo lugar su esencia. En tercer lugar, tendríamos el acto, entendido por Zubiri como la plenitud de la realidad (o del ser) de la cosa. Y todavía quedaría abierta la cuestión de la posible actualidad intelectiva de este acto, que es lo que desembocaría en la verdad como cuarto sentido del ser.

No cabe duda de que se trata de una exposición coherente de Aristóteles, y que encaja bien con la dirección general de la filosofía de Zubiri. En esta perspectiva, ¿cómo se puede entender, en la filosofía de Zubiri, la mismidad de acto en la inteligencia, de la que nos hablaba Aristóteles? Si el acto es la plenitud de la realidad de algo, podríamos pensar que, en el caso del sentir, Aristóteles nos estaría diciendo que la plenitud del ser del acto sensorial sería la plenitud del ser de la cosa sentida. En el caso de un color, la plenitud del ser del acto visual como acto completamente ejecutado sería la plenitud del ser del color mismo. Pero esta interpretación no parece tener mucho sentido. Como el mismo Aristóteles nos ha dicho, el acto del sentir y el acto de lo sentido es uno y el mismo, pero su ser es distinto ${ }^{39}$. Esta afirmación aristotélica no tendría ningún sentido si el acto fuera para Aristóteles la plenitud de la realidad (o del ser) de una cosa, como pretende Zubiri. Esto es algo que el mismo Zubiri entrevió en Sobre la esencia cuando, al exponer la mismidad de acto en Aristóteles, tuvo que admitir dos sentidos distintos de acto en Aristóteles. Dicho en otros términos: el acto aristotélico, la enérgeia, no puede significar para Aristóteles la plenitud del ser de algo. Al menos en este caso, la enérgeia aristotélica tiene que tener otro sentido.

35 Cf. ARISTóteles, Metafísica 1027 b 17- 34.

36 Cf. ibid., 1051 a $34-1052$ a 11.

37 Cf. ARistóteles, Ética a Nicómaco 1139 b 12-13.

38 La ousiología de Aristóteles sería, «en último término, energuiología» cf. VIGo, A.G., Aristóteles. Una introducción. Santiago de Chile: Instituto de Estudios de la Sociedad, p. 178.

39 Cf. ARISTóteles, Sobre el alma 425 b 27. 
¿En qué consiste entonces, para Aristóteles, la diferencia entre el acto (común entre el sentir y lo sentido) y el ser, distinto entre ambos? En el caso de la mismidad de acto en el sentir, Aristóteles piensa con el modelo del movimiento ${ }^{40}$. El movimiento es, para Aristóteles, un acto, justamente el acto de lo que está en potencia, en cuanto que está en potencia ${ }^{41}$. En el caso del sentir, tenemos por un lado nuestra potencia o capacidad de sentir, y por el otro lado la potencia que tiene la cosa de ser sentida. Se trata de potencias distintas, en entes distintos. Por eso dice Aristóteles que su ser es distinto. Sin embargo, hay un mismo acto, que es el acto de sentir y el acto de ser sentido. Para Aristóteles es algo semejante a lo que sucede en otros procesos. Por ejemplo, el acto de empujar una mesa que realiza un agente es el mismo acto en que esa mesa es movida. Pero su ser es distinto. $\mathrm{O}$ el acto de enseñar es el mismo acto que el de ser enseñado, pero obviamente el ser de los profesores y de los alumnos es distinto ${ }^{42}$.

En el caso de la inteligencia nos encontramos con una situación algo distinta, en el sentido de que, para Aristóteles, el acto del entendimiento agente está libre de potencias, es acto por su propia entidad (ousía) ${ }^{43}$, de modo que en el entendimiento no hay diferencia entre su acto y su ser. Sin embargo, en la cosa inteligida es posible distinguir entre el ser de la cosa y el acto de ser inteligida. La cosa tiene la potencia de ser inteligida, con independencia de que de hecho lo sea. De nuevo, la identidad entre la inteligencia y la cosa es una identidad de acto, pero no de ser. La inteligencia se identifica con las cosas solamente en cuanto que estas están inteligidas. Es lo que Zubiri, a la altura de Sobre la esencia, explicaba acudiendo a la intencionalidad como un modo de ser: el modo de ser de las cosas intelectivas. En cualquier caso, tampoco aquí el acto aristotélico puede ser interpretado como plenitud de la realidad de algo. Habría que admitir, como hace Zubiri en Sobre la esencia, que a veces el acto aristotélico tiene otro sentido.

Y este sentido, según la terminología del Zubiri maduro, tendría que ser el de actualidad, distinta de la plenitud de la realidad de la cosa. En el acto intelectivo tendríamos una actualidad común, en el sentido de que la actualidad de de la cosa es la misma que la actualidad del propio acto intelectivo. Pero tendríamos dos realidades distintas. Veamos esto más detenidamente.

\section{LA ACTUALIDAD COMÚN}

Desde el punto de vista sistemático, Zubiri entiende que la esencia de la intelección sentiente consiste en actualidad, y que esta actualidad es una actualidad

40 Cf. ARistóteles, Sobre el alma 425 b 26 - 426 a 15.

41 Cf. Aristóteles, Física 201 b 3-4.

42 Cf. Aristóteles, Metafísica 1066 a 25-34; Física 202 b 19-22.

43 Cf. ARIstóteles, Sobre el alma 430 a 18. 
unitaria, o común, pues una misma actualidad es la actualidad de la cosa inteligida y la actualidad del acto de intelección ${ }^{44}$.

Aquí tenemos entonces dos niveles distintos. En primer lugar, está el nivel de la "actuidad», en el que encontramos dos «actuidades» distintas. Una es la «actuidad» en el sentido de la plenitud de la realidad de las cosas reales allende la aprehensión. Y, por otro lado, estaría la «actuidad» del acto intelectivo como acto ejecutado. Por supuesto, para Zubiri se trata de dos realidades distintas, de dos «actuidades» distintas, que convergen en el acto de intelección. Por supuesto, en este nivel, las consideraciones sobre las «condiciones de posibilidad» del acto intelectivo a partir de estas dos «actuidades» distintas se moverían en el ámbito explicativo o metafísico, que no es el que a Zubiri le interesa primeramente.

En segundo lugar, en el nivel de la actualidad, tenemos una sola actualidad común. Es la actualidad de la cosa, que es la misma actualidad que la del acto intelectivo. Ahora bien, la actualidad del acto intelectivo tiene que ser, como tal actualidad, la actualidad de algo real. Y es que, para Zubiri, el propio acto intelectivo es algo real. Como él mismo afirma: «mi propio acto de intelección sentiente es un acto real, es una realidad. Y esta realidad está actualizada con la realidad de la cosa en la misma actualidad que ésta ${ }^{45}$.

Ahora bien, ¿en qué sentido es el acto «real»? El acto no es una cosa más, que se actualice como algo «de suyo». Y Zubiri es bien consciente de esto. El modo de actualidad del acto es radicalmente distinto de las cosas. Según Zubiri, el acto se actualiza «No ciertamente como una cosa más, sino en esa forma que expresa el gerundio español "estoy sintiendo" ${ }^{46}$. Esta apelación al gerundio español tiene una honda tradición en la obra de Zubiri. En esta expresión, como puede verse, se emplea el verbo estar, que es para Zubiri precisamente el verbo en el que se expresa la actualidad. La actualidad común de los actos intelectivos consistiría en que todo acto envuelve, no sólo la actualidad de las cosas que intelijo, sino también este momento de «estar sintiendo» o "estar inteligiendo» las cosas.

Precisamente con esta apelación al gerundio Zubiri había criticado a Descartes en Los problemas fundamentales de la metafísica occidental. Para Zubiri, toda la fuerza del cogito ergo sum radica, no en el cogitar, no en el pensamiento, sino en que, en el cogito, «estoy» pensando. Como dice Zubiri,

«El carácter pensante pasa a segundo plano, y lo que nos queda en primer plano es ese "estar": yo pienso que estoy pensando. Justamente en el "estar" es donde estriba la actualidad del pensamiento, y por consiguiente, su carácter mismo de realidad. La realidad del pensamiento, y no solamente su dimensión de verdad, es lo que constituye la fuerza inicial y radical del cogito ${ }^{47}$.

${ }^{44}$ Cf. Zubiri, X. (1980), Inteligencia sentiente. Inteligencia y realidad, op. cit., pp. 133-188.

45 Cf. ibíd., p. 157.

46 Cf. ibíd., p. 161.

47 Cf. ZuBiRI, X. (1995), Los problemas fundamentales de la metafísica occidental, op. cit., p. 134. 
No vamos a entrar aquí en la validez de la interpretación zubiriana de Descartes, que está basada en la expresión ego cogito me cogitare, la cual propiamente no se encuentra en las obras del filósofo francés. Zubiri la recibió de Heidegger, que también entiende de esta manera libre el cogito cartesiano ${ }^{48}$. Otras expresiones auténticas de Descartes, como el videre videor o el cogito me cogitare, posiblemente serían más justas desde un punto de vista histórico, y abrirían otras posibilidades de lectura de su obra ${ }^{49}$.

En cualquier caso, la idea de Zubiri según la cual la actualidad propia de los actos consiste en un «estar sintiendo» o en un «estar pensando» plantea al menos dos importantes cuestiones, que tenemos que ver con detenimiento:

a) Hay una dificultad referente a la descripción del acto intelectivo en términos de actualidad común. Y es que esta conceptualización aboca a un regreso al infinito. En el acto intelectivo, se actualizaría la cosa, pero también el propio acto intelectivo, el cual incluye de nuevo la cosa y el acto intelectivo, etc. La actualidad común sería actualidad de la cosa y del acto intelectivo, el cual a su vez sería actualidad de la cosa y del acto intelectivo.

Claro está que esta dificultad no es la más importante, porque enuncia un problema meramente lógico, mientras que la actualidad común se sitúa en un plano anterior a la lógica. La mismidad de actualidad no es una identidad lógica, sino una identidad de actualidad. Como dice Zubiri:

"No se trata de una identidad lógica; se trata precisamente de la identidad del estar; real y efectivamente es estando como "estoy" pensando y como "estoy" viendo que "estoy" pensando. El acceso del hombre a sí mismo no es una cuestión de intuición; es la condición de una realidad [...], de un estar, que no puede estar —en este caso pensado— más estando intuyendo y viendo que efectivamente está pensando» ${ }^{50}$.

b) Ahora bien, en esta identidad de actualidad, ¿podemos decir que tenemos la actualidad de un acto? No cabe duda de que el «estar inteligiendo» expresa una actualidad. Pero el «estoy» de esta actualidad alude más bien a mi propia realidad completa. La plenitud de la realidad actualizada no es la actualidad de un acto, es la actualidad de mi realidad personal. Cuando inteligimos algo, no sólo se actualiza la cosa inteligida, sino también mi propia realidad.

Cuando decimos que «estamos pensando» o que «estamos sintiendo» no nos referimos al carácter «atingente» del acto respecto a su objeto, como a veces indica Zubiri ${ }^{51}$. Lo que indicamos es que, al pensar, o al sentir, o al realizar

48 Cf. Heidegger, M. (1961), Nietzsche, vol. 2. Pfullingen: Neske, p. 148.

49 Puede verse Henry, M. (1990), Phénoménologie matérielle. Paris: Presses Universitaires de France, y Henry, M. (2000), Incarnation. Une philosophie de la chair. Paris: Éditions du Seuil.

50 Cf. Zubiri, X. (1995), Los problemas fundamentales de la metafísica occidental, op. cit., p. 135.

51 Cf. ibid., p. 135. 
cualquier otro acto intelectivo, mi propia realidad esta co-actualizada en el acto intelectivo. Si estoy pensando, es que mi propia realidad está presente en el acto mismo de pensar. La actualidad del «estoy sintiendo» es la actualidad de mi realidad corpórea, y no la actualidad de mi acto de intelección.

El problema está en que el acto de intelección no aparece en las notas de mi realidad actualizada. El acto intelectivo ciertamente acontece como acto, y como acto atingente en el sentido de estar "tocando" las cosas actualizadas, y estar totalmente determinado por ellas. Pero el acto mismo no parece actualizarse. Por más que sea el responsable de la actualización de la cosa, y de la actualización de mi propia realidad, el acto mismo no se actualiza.

\section{INTENTO DE SOLUCIÓN}

Yendo más allá de la literalidad del planteamiento de Zubiri, podríamos encontrar una vía de solución si entendemos que el acto intelectivo no consiste propiamente en una realidad dotada de actualidad intelectiva, sino que su estatuto ontológico es distinto. Dicho en otros términos: el acto no consistiría en «actuidad», en la plenitud de la realidad de algo, sino en actualización. La actualización no responde a una consideración explicativa sobre la ejecución de los actos por un sujeto, sino que es un modo de describir el hecho de que las cosas se manifiestan en nuestros actos. Desde este punto de vista, el acto sería la actualización misma de las cosas.

La actualización misma no es algo que se actualice. Como tal actualización, es invisible. Y, si la actualización no se actualiza, tendríamos que decir que, propiamente, no hay una actualidad común entre el acto y la cosa. Ciertamente, hay una actualidad común entre mi propia realidad y la realidad de la cosa. Pero el acto mismo no es otra realidad más, sino la actualización de la cosa y, por supuesto, también la actualización de mi propia realidad. Los actos no pertenecen al orbe de la realidad, sino a un ámbito ontológico distinto.

En la actualización de la realidad (incluyendo la actualización de mi propia realidad) la mismidad de actualidad no abarca a los actos. Los actos no se actualizan. Ahora bien, si los actos son la actualización de las cosas, hay que afirmar que los actos están completamente determinados por las cosas que se actualizan. La única actualidad que tienen los actos es la actualidad de lo que se actualiza, incluyendo las cosas inteligidas y la propia realidad.

El acto no se actualiza, sino que es la actualización misma. Por eso no sólo nos evitamos cualquier regreso al infinito, sino que también podemos hablar, con Heidegger y con Zubiri, de un «darse». Y de un «darse» donde aquello que se da configura plenamente al acto, porque la actualidad es intrínseca al acto mismo entendido como actualización. En este sentido, la apelación de Zubiri a Aristóteles para complementar a Heidegger sigue teniendo un perfecto sentido, y no nos aboca a ninguna «metafísica» de acto en términos de presencia, que por cierto no son los de Aristóteles. 


\subsection{Aristóteles}

De este modo podemos integrar el planteamiento aristotélico. Cuando Aristóteles quiere proporcionarnos una noción de lo que sea el acto, la enérgeia, nos dice literalmente que el acto es «el surgir la cosa» ${ }^{52}$. Aristóteles dice $h y$ párkhein, es decir, un hypo-árkhein, que equivale literalmente a sub-regere, es decir, al castellano «surgir». En lugar de traducir este hypárkhein con términos anacrónicos, como "existir», sería más conveniente hablar de "surgir» o, si se quiere, en términos zubirianos, de «actualización». Los actos no serían ni «actuidad» ni actualidad, sino el actualizarse, el surgir de las cosas.

Precisamente por ello se nos hace ahora clara la tesis aristotélica de que, en la mismidad de acto, el ser de lo sentido y el ser de la sensación son distintos. En términos más generales, también podríamos decir, que el ser de lo inteligido y el ser de la intelección son distintos. La mismidad de acto sería, propiamente, la unidad de una sola y única actualidad, la actualidad de la cosa, que configura intrínsecamente al acto mismo, por consistir el acto en actualización. Y, sin embargo, el ser de los actos no es el mismo que el ser de las cosas inteligidas en esos actos.

\subsection{Heidegger}

Aquí es donde propiamente podemos hablar de una "diferencia ontológica», que nos conduce a integrar el planteamiento de Heidegger. Respecto a Heidegger, podemos decir que el «darse» (es gibt) se nos muestra ahora como un «surgir» o un «actualizarse» de las cosas. Este surgir designa un ámbito ontológico propio, distinto de la realidad de las cosas actualizadas, que hace pensable el camino hacia el Ereignis. Se trata de un camino que nos lleva claramente más allá del ser. No se trata de un ser arcaico (Seyn) ni de un ser olvidado, sino formalmente algo que está más allá del ser. La discusión del «darse» rebasa la disputa sobre la prioridad de la realidad sobre el ser, porque nos lleva más allá de la realidad y más allá del ser.

No se trata aquí de discutir qué es primero, la actualidad o la desvelación, la actualidad o el «darse», la actualidad o la actualización. De lo que se trata es simplemente de distinguir entre dos ámbitos diversos. Uno es el ámbito de lo que está presente. Otro es el ámbito del llegar a la presencia.

Este ámbito radicalmente distinto de la presencia se sitúa en los actos. Los actos, la enérgeia aristotélica, el hypárkhein, no se refieren a la presencia de lo actualizado, sino a la actualización misma ${ }^{53}$. Por eso los actos, como un surgir de las cosas, no aparecen, no son actuales. La única actualidad es la actualidad de las cosas actualizadas, porque los actos consisten en actualización. Por otra parte, los actos

52 Ésti dè enérgeia tò hypárkhein tò prâgma, en ArIstóteles, Metafísica 1048 a 30-31.

53 Lamentablemente, Heidegger interpretó el hypárkhen aristotélico como presencialidad, y no como un surgir, cf. HeIDEgGer, M. (1976), Logik. Die Frage nach der Wahrheit. Frankfurt a. m.: Vittorio Klostermann, p. 132, 
no son la fórmula de un subjetivismo, porque no son tomados kathà dýnamin, como actos de la conciencia o actos del sujeto, sino por sí mismos, como actos.

\subsection{Zubiri}

En este punto reside una de las grandes aportaciones del pensamiento de Zubiri. Zubiri ha sido capaz de vincular el análisis de los actos, de raigambre fenomenológica, con la recuperación de la enérgeia aristotélica. Con ello ha sacado el análisis de los actos del paradigma subjetual de la filosofía moderna.

Y, sin embargo, el análisis kath' enérgeian no nos aboca a ninguna forma de deshumanización. Todo lo contrario: en la medida en que los actos se nos muestran como un ámbito ontológico propio, distinto de las realidades actualizadas, se nos abren interesantísimas posibilidades de desarrollar una antropología que pueda mostrar la irreductibilidad radical de lo personal a lo cósico.

Al mismo tiempo, en el análisis de los actos, la alteridad extrema que Zubiri encuentra en ellos, la alteridad de la realidad, se mantiene en toda su validez. La alteridad de las cosas inteligidas no es solamente la alteridad «intencional» de un sentido. Las cosas se actualizan en nuestros actos como radicalmente otras. Respecto a esta «reidad» de las cosas, sigue siendo posible decir con $\mathrm{Zu}-$ biri que el ser es ulterior a la realidad.

Ahora bien, es interesante observar que la «realidad», desde este punto de vista, no se nos presente primeramente como formalidad, como una especie de «gota de aceite» (en palabras del mismo Zubiri) ${ }^{54}$, que se extiende de unas cosas a otras. La alteridad radical de las cosas actualizadas respecto a su actualización consiste posiblemente en algo mucho más sencillo y radical. Y es que las cosas actualizadas son siempre radicalmente distintas de los actos, porque los actos no son cosas, ni se actualizan como realidades. La radical alteridad de la realidad se nos presenta más bien como una «diferencia ontológica» (o «ultra ontológica») entre la actualización y lo actualizado, entre el surgir y lo que surge.

Fundación Xavier Zubiri

Antonio GonZález glez.fdez.antonio@gmail.com

[Artículo aprobado para publicación en este número extraordinario en noviembre de 2014]

54 Cf. ZuBIRI, X. (1980), Inteligencia sentiente. Inteligencia y realidad, op. cit., p. 118. 
